\title{
JOINT CONTINUITY OF INJECTIVE TENSOR PRODUCTS OF VECTOR MEASURES IN BANACH LATTICES
}

\author{
JUN KAWABE \\ (Received 15 February 2001; revised 7 November 2001)
}

Communicated by A. Pryde

\begin{abstract}
It is shown that the injective tensor product of positive vector measures in certain Banach lattices is jointly continuous with respect to the weak convergence of vector measures. This result is obtained by a diagonal convergence theorem for injective tensor integrals. Our approach to this problem is based on Bartle's bilinear integration theory.
\end{abstract}

2000 Mathematics subject classification: primary 28A33, 28B05; secondary 46A11, 46A32.

Keywords and phrases: positive vector measure, injective tensor product of vector measures, weak convergence of vector measures, Banach lattice, injective tensor integral.

\section{Introduction}

In 1967, Duchon and Kluvánek [8] introduced the notion of tensor products of vector measures which we describe in our setting: Let $(\Omega, \mathscr{A})$ and $(\Gamma, \mathscr{B})$ be measurable spaces. Let $X$ and $Y$ be Banach spaces, and $X \widetilde{\otimes} Y$ the injective tensor product of $X$ and $Y$ (see [7, Chapter VIII]). For any vector measures $\mu: \mathscr{A} \rightarrow X$ and $\nu: \mathscr{B} \rightarrow Y$, there always exists a unique vector measure $\mu \widetilde{\otimes} v: \mathscr{A} \times \mathscr{B} \rightarrow X \widetilde{\otimes} Y$, which is called the injective tensor product of $\mu$ and $\nu$, such that $(\mu \widetilde{\otimes} \nu)(A \times B)=\mu(A) \otimes \nu(B)$ for all $A \in \mathscr{A}$ and $B \in \mathscr{B}$. On the other hand, Dekiert [6] recently introduced the notion of weak convergence of Banach space-valued measures, which is a natural generalization of the weak convergence of probability measures, and studied its properties (see also $[19,22])$.

This work has been supported by Grant-in-Aid for General Scientific Research No. 11640160, the Ministry of Education, Science, Sports and Culture, Japan.

(C) 2003 Australian Mathematical Society 1446-7887/03 \$A2.00+0.00 
Let $S$ and $T$ be topological spaces. In this paper, we prove that if nets $\left\{\mu_{\alpha}\right\}$ and $\left\{v_{\alpha}\right\}$ of positive vector measures on $S$ and $T$ with values in Banach lattices $X$ and $Y$ converge weakly to $\mu$ and $\nu$, respectively, then the injective tensor product $\mu_{\alpha} \widetilde{\otimes} \nu_{\alpha}$ converges weakly to $\mu \widetilde{\otimes} v$ under some additional assumptions.

The injective tensor product of two probability measures is just the usual product measure, so that its joint continuity is well known in the case that $S$ and $T$ are separable metric spaces [3, Theorem 3.2], and more generally completely regular spaces [24, Proposition I.4.1]. It was also shown in [5, Corollary to Theorem 1] that the convolution of probability measures on an arbitrary topological group is jointly continuous.

In a recent paper [14], we studied this kind of problem for vector measures with values in certain nuclear spaces. It is shown in [14, Theorems 5 and 7] that the weak convergence of a net of injective tensor products of uniformly bounded nuclear space-valued vector measures follows from that of the corresponding net of real product measures. The way of proving the above result is essentially based on a finite dimensional feature of nuclear spaces, that is, the weak topology coincides with the original topology on every bounded subset of any barreled, quasi-complete nuclear space. Therefore, the same method may not apply to the case of vector measures with values in Banach spaces. The purpose of this paper is to show that this type of joint continuity of product measures remains true for the injective tensor products of positive vector measures in certain Banach lattices; see Theorem 5.4. Our approach to this problem is based on the Bartle bilinear vector integration [1].

In Section 2, we formulate some notation and results which are needed in the sequel. In Section 3, we give some technical results of the injective tensor integrals, which are the Bartle bilinear integrals with respect to the injective tensor product. In Section 4 , we give a diagonal convergence theorem for the injective tensor integrals, which is not only crucial to prove our main result, but seems to be of some interest. Using this diagonal convergence theorem, it is shown in Section 5 that the joint continuity of the injective tensor products remains true for positive vector measures with values in Banach lattices under some additional assumptions.

All the topological spaces and uniform spaces in this paper are Hausdorff, and the scalar fields of Banach spaces are taken to be the field $\mathbb{R}$ of all real numbers. Denote by $\mathbb{N}$ the set of all natural numbers.

\section{Preliminaries}

Let $X$ be a Banach space with norm $\|\cdot\|$ and $X^{*}$ the topological dual of $X$. Let $\boldsymbol{B}_{X}$. denote the closed unit ball of $X^{*}$. Let $(\Omega, \mathscr{A})$ be a measurable space. A $\sigma$-additive set function $\mu: \mathscr{A} \rightarrow X$ is called a vector measure. The semivariation of $\mu$ is the set 
function $\|\mu\|(A)=\sup \left\{\left|x^{*} \mu\right|(A): x^{*} \in B_{X^{*}}\right\}$, where $\left|x^{*} \mu\right|(\cdot)$ is the total variation of $x^{*} \mu$. Then $\|\mu\|(\Omega)<\infty[2$, Lemma 2.2].

Let $\mu: \mathscr{A} \rightarrow X$ be a vector measure. Denote by $\chi_{A}$ the indicator function of a set $A$. A $\mu$-null set is a set $E \in \mathscr{A}$ for which $\|\mu\|(E)=0$; the term $\mu$-almost everywhere refers to the complement of a $\mu$-null set. Given an $\mathscr{A}$-simple function of the form $f=\sum_{k=1}^{m} a_{k} \chi_{A_{k}}$ with $a_{1}, \ldots, a_{m} \in \mathbb{R}, A_{1}, \ldots, A_{m} \in \mathscr{A}, m \in \mathbb{N}$, define its integral $\int_{A} f d \mu$ over a set $A \in \mathscr{A}$ by $\int_{A} f d \mu=\sum_{k=1}^{m} a_{k} \mu\left(A_{k} \cap A\right)$. An $\mathscr{A}$-measurable function $f: \Omega \rightarrow \mathbb{R}$ is said to be $\mu$-integrable if there exists a sequence $\left\{f_{n}\right\}$ of $\mathscr{A}$-simple functions converging $\mu$-almost everywhere to $f$ such that the sequence $\left\{\int_{A} f_{n} d \mu\right\}$ converges in the norm of $X$ for each $A \in \mathscr{A}$. This limit $\int_{A} f d \mu$ does not depend on the choice of such simple functions $f_{n}, n \in \mathbb{N}$. By the Orlicz-Pettis theorem [7, Corollary I.4.4], the indefinite integral $A \mapsto \int_{A} f d \mu$ is $\sigma$-additive. Every bounded, $\mathscr{A}$-measurable function $f: \Omega \rightarrow \mathbb{B}$ is $\mu$-integrable, and $\left\|\int_{A} f d \mu\right\| \leq \sup _{\omega \in A}|f(\omega)| \cdot\|\mu\|(A)$ for each $A \in \mathscr{A}[2$, Theorem 2.6]. For further properties of this integral see $[2,10,17,18]$.

We define several notions of regularity for vector measures on a topological space. Let $S$ be a topological space and $\mathscr{B}(S)$ the $\sigma$-algebra of all Borel subsets of $S$. Let $\mu: \mathscr{B}(S) \rightarrow X$ be a vector measure. We say that $\mu$ is Radon if for every $\varepsilon>0$ and $A \in \mathscr{B}(S)$ there exists a compact subset $K$ of $A$ such that $\|\mu\|(A-K)<\varepsilon$, and it is tight if this condition is satisfied for $A=S$. We say that $\mu$ is $\tau$-smooth if, for any increasing net $\left\{G_{\alpha}\right\}$ of open subsets of $S$ with $G=\bigcup_{\alpha} G_{\alpha}$, we have $\lim _{\alpha}\|\mu\|\left(G-G_{\alpha}\right)=0$. It follows that $\mu$ is Radon (respectively tight, $\tau$-smooth) if and only if, for each $x^{*} \in X^{*}$, the real measure $x^{*} \mu$ is Radon (respectively tight, $\tau$-smooth). In fact, this is a consequence of the Rybakov theorem [7, Theorem IX.2.2], which ensures that there exists $x_{0}^{*} \in X^{*}$ for which $x_{0}^{*} \mu$ and $\mu$ are mutually absolutely continuous.

The following result can be proved as in the case of scalar measures; see for example [24, Proposition I.3.2].

PROPOSITION 2.1. Let $S$ be a topological space and $X$ a Banach space. Let $\mu: \mathscr{B}(S) \rightarrow X$ be a $\tau$-smooth vector measure. Let $\left\{f_{\alpha}\right\}$ be a uniformly bounded decreasing net of upper semicontinuous non-negative functions on $S$. If $f=\lim _{\alpha} f_{\alpha}$ is the pointwise limit of $f_{\alpha}$, then $f_{\alpha}$ 's and $f$ are all $\mu$-integrable and $\lim _{\alpha} \int_{S} f_{\alpha} d \mu=$ $\int_{S} f d \mu$.

\section{Some properties of injective tensor integrals}

In this section, we define the Bartle bilinear integration in our setting: Let $X$ and $Y$ be Banach spaces. Denote by $X \widetilde{\otimes} Y$ the injective tensor product of $X$ and $Y$; see $[7$, Chapter VIII]. Let $(\Omega, \mathscr{A})$ be a measurable space. Let $\varphi: \Omega \rightarrow X$ be a vector 
function and $\nu: \mathscr{A} \rightarrow Y$ a vector measure. Given an $X$-valued simple function $\varphi=\sum_{k=1}^{m} x_{k} \chi_{A_{k}}$ with $x_{1}, \ldots, x_{m} \in X, A_{1}, \ldots, A_{m} \in \mathscr{A}, m \in \mathbb{N}$, define its integral $\int_{A} \varphi \widetilde{\otimes} d v$ over a set $A \in \mathscr{A}$ by $\int_{A} \varphi \widetilde{\otimes} d \nu=\sum_{k=1}^{m} x_{k} \otimes \nu\left(A_{k} \cap A\right)$. We say that $\varphi$ is $\nu$-measurable if there exists a sequence $\left\{\varphi_{n}\right\}$ of $X$-valued simple functions converging $\nu$-almost everywhere to $\varphi$. The function $\varphi$ is said to be $\nu$-integrable in the sense of Bartle if there exists a sequence $\left\{\varphi_{n}\right\}$ of $X$-valued simple functions converging $\nu$-almost everywhere to $\varphi$ such that the sequence $\left\{\int_{A} \varphi_{n} \widetilde{\otimes} d \nu\right\}$ converges in the norm of $X \widetilde{\otimes} Y$ for each $A \in \mathscr{A}$. This limit $\int_{A} \varphi \widetilde{\otimes} d \nu$ does not depend on the choice of such $X$-valued simple functions $\varphi_{n}, n \in \mathbb{N}$, and the indefinite integral $A \rightarrow \int_{A} \varphi \tilde{\otimes} d v$ is an $X \widetilde{\otimes} Y$-valued vector measure on $\mathscr{A}$.

For simplicity, we say that the $\varphi$ is $\nu$-integrable if it is $\nu$-integrable in the sense of Bartle. The integral $\int_{A} \varphi \widetilde{\otimes} d \nu$ is called the injective tensor integral of $\varphi$ over $A$ with respect to $\nu$. See a recent paper [11] for further properties of injective tensor integrals such as some characterizations of integrable functions and the general Fubini theorem.

In the following, we prepare some technical properties of injective tensor integrals which will be used in Section 4. The proof of the following lemma is obvious and will be omitted.

LEMMA 3.1. Let $(\Omega, \mathscr{A})$ be a measurable space. Let $X$ and $Y$ be Banach spaces. Let $\nu: \mathscr{A} \rightarrow Y$ be a vector measure and $f: \Omega \rightarrow \mathbb{R}$ a v-integrable, $\mathscr{A}$-measurable function. Then, given $x \in X$, the vector function $f x: \Omega \rightarrow X$ defined by $(f x)(\omega)=$ $f(\omega) x$ for $\omega \in \Omega$ is $\nu$-integrable and $\int_{A}(f x) \widetilde{\otimes} d v=x \otimes \int_{A} f d v$ for each $A \in \mathscr{A}$.

When the Banach space $X$ is equipped with the additional structure of a Banach lattice, we may introduce the notion of positivity for vector measures. We say that a vector measure $\mu: \mathscr{A} \rightarrow X$ is positive if $\mu(A) \geq 0$ for every $A \in \mathscr{A}$. By [22, Lemma 1.1], for every positive vector measure $\mu$, we have $\|\mu\|(A)=\|\mu(A)\|$ for all $A \in \mathscr{A}$. Further, it is easy to verify that for any $\mu$-integrable, $\mathscr{A}$-measurable real functions $f$ and $g$ with $|f| \leq g \mu$-almost everywhere, we have

$$
\left|\int_{\Omega} f d \mu\right| \leq \int_{\Omega}|f| d \mu \leq \int_{\Omega} g d \mu \quad \text { and } \quad\left\|\int_{\Omega} f d \mu\right\| \leq\left\|\int_{\Omega}|f| d \mu\right\| \leq\left\|\int_{\Omega} g d \mu\right\| .
$$

These facts greatly facilitate the analysis of positive vector measures and are used frequently without saying these explicitly in this paper. For further properties of positive vector measures on metric spaces, see $[19,22]$. For other applications of positivity of vector measures, see [15]. We refer the reader to the book of Schaefer [20] for the basic theory of Banach lattices.

Proposition 3.2. Let $(\Omega, \mathscr{A})$ be a measurable space. Let $X$ be a Banach space and $Y$ a Banach lattice. Let $\nu: \mathscr{A} \rightarrow Y$ be a positive vector measure. If an $\mathscr{A}$-measurable function $f: \Omega \rightarrow \mathbb{B}$ is $\nu$-integrable and a vector function $\varphi$ : 
$\Omega \rightarrow X$ is bounded and $\nu$-measurable, then the vector function $f \varphi: \Omega \rightarrow X$ defined by $(f \varphi)(\omega)=f(\omega) \varphi(\omega), \omega \in \Omega$, is v-integrable, and $\left\|\int_{A}(f \varphi) \widetilde{\otimes} d \nu\right\| \leq$ $\sup _{\omega \in \Omega}\|\varphi(\omega)\| \cdot\left\|\int_{A}|f| d v\right\|$ for each $A \in \mathscr{A}$.

PROOF. The function $\varphi$ is $\nu$-integrable by [1, Theorem 4], and hence we can find a sequence $\left\{\varphi_{n}\right\}$ of $X$-valued simple functions on $\Omega$ converging $\nu$-almost everywhere to $\varphi$ such that $\left\|\varphi_{n}(\omega)\right\| \leq\|\varphi(\omega)\|$ for all $\omega \in \Omega$ and $n \in \mathbb{N}$ (see, for instance, [25, Theorem 2.6]).

Fix $A \in \mathscr{A}$ and $n \in \mathbb{N}$ and put $\|\varphi\|_{\infty}=\sup _{\omega \in \Omega}\|\varphi(\omega)\|$. The function $f \varphi_{n}$ which is $\nu$-integrable by Lemma 3.1 satisfies

$$
\left\|\int_{A}\left(f \varphi_{n}\right) \widetilde{\otimes} d \nu\right\|=\sup _{\boldsymbol{x}^{*} \in \boldsymbol{B}_{\boldsymbol{X}^{*}}}\left\|\int_{A} f \cdot\left(x^{*} \varphi_{n}\right) d \nu\right\| ;
$$

see [11, page 327]. Since $\left|f(\omega)\left(x^{*} \varphi_{n}\right)(\omega)\right| \leq\|\varphi\|_{\infty}|f(\omega)|$ for all $x^{*} \in B_{X^{*}}$, the positive measure $\nu$ satisfies $\left|\int_{A} f \cdot\left(x^{*} \varphi_{n}\right) d \nu\right| \leq\|\varphi\|_{\infty} \int_{A}|f| d \nu$, which implies that

$$
\sup _{x^{*} \in B_{X^{*}}}\left\|\int_{A} f \cdot\left(x^{*} \varphi_{n}\right) d \nu\right\| \leq\|\varphi\|_{\infty}\left\|\int_{A}|f| d \nu\right\| .
$$

Since $f \varphi_{n}$ converges $\nu$-almost everywhere to $f \varphi$, it follows from (3.1), (3.2) and [1, Theorem 10] that $f \varphi$ is $\nu$-integrable and $\int_{A}\left(f \varphi_{n}\right) \widetilde{\otimes} d \nu \rightarrow \int_{A}(f \varphi) \widetilde{\otimes} d \nu$. This together with (3.1) and (3.2) gives the required inequality.

Let $T$ be a topological space and $\mathscr{B}(T)$ the $\sigma$-algebra of all Borel subsets of $T$. Let $C(T, X)$ denotes the Banach space of all bounded continuous functions $\varphi: T \rightarrow X$ with the norm $\|\varphi\|_{\infty}=\sup _{t \in T}\|\varphi(t)\|$. We write $C(T)=C(T, \mathbb{R})$.

PROPOSITION 3.3 ([26, Theorem 1.6]). Let $T$ be a topological space. Let $X$ and $Y$ be Banach spaces. Let $\nu: \mathscr{B}(T) \rightarrow Y$ be a tight vector measure and $\varphi \in C(T, X)$. Then, $\varphi$ is $v$-integrable, and $\left\|\int_{A} \varphi \widetilde{\otimes} d v\right\| \leq \sup _{t \in A}\|\varphi(t)\| \cdot\|\nu\|(A)$ for all $A \in \mathscr{B}(T)$.

ProOF. By [1, Theorem 4] we have only to prove that $\varphi$ is $\nu$-measurable. Take an increasing sequence $\left\{K_{n}\right\}_{n=1}^{\infty}$ of compact subsets of $T$ such that $T-\bigcup_{n=1}^{\infty} K_{n}$ is $\nu$-null. Since $\varphi\left(K_{n}\right)$ is compact for all $n \in \mathbb{N}$, the set $\varphi\left(\bigcup_{n=1}^{\infty} K_{n}\right)$ is $\sigma$-compact, and hence separable. Apply the Pettis measurability theorem [7, Theorem II.1.2] to conclude that $\varphi$ is $\mu$-measurable.

\section{A diagonal convergence theorem for injective tensor integrals}

Let $T$ be a uniform space with the uniformity $\mathscr{U}_{T}$. Let $X$ be a Banach space. Denote by $U(T, X)$ the Banach space of all bounded uniformly continuous functions 
$\varphi: T \rightarrow X$ with the norm $\|\varphi\|_{\infty}=\sup _{t \in T}\|\varphi(t)\|$. We write $U(T)=U(T, \mathbb{R})$. Let $Y$ be a Banach lattice. Denote by $\mathscr{M}^{+}(T, Y)$ the set of all positive vector measures $\nu: \mathscr{B}(T) \rightarrow Y$, and denote by $\mathscr{M}_{t}^{+}(T, Y)$ the set of all $\nu \in \mathscr{M}^{+}(T, Y)$ which are tight.

In this section, we give a diagonal convergence theorem for injective tensor integrals with respect to positive vector measures. The following theorem is not only crucial to prove our main results, that is Theorem 5.3 and Theorem 5.4, but seems to be of some interest.

THEOREM 4.1. Let $T$ be a uniform space with the uniformity $\mathscr{U}_{T}$. Let $X$ be a Banach space and $Y$ a Banach lattice. Consider a net $\left\{\varphi_{\alpha}\right\} \subset U(T, X)$ and $\varphi \in U(T, X)$ satisfying the following conditions:

(i) $\varphi_{\alpha}(t) \rightarrow \varphi(t)$ for every $t \in T$;

(ii) $\left\{\varphi_{\alpha}\right\}$ is uniformly bounded, that is, $\sup _{\alpha}\left\|\varphi_{\alpha}\right\|_{\infty}<\infty$; and

(iii) $\left\{\varphi_{\alpha}\right\}$ is uniformly equicontinuous on $T$, that is, for any $\varepsilon>0$, there exists $V \in \mathscr{U}_{T}$ such that $\sup _{\alpha}\left\|\varphi_{\alpha}(t)-\varphi_{\alpha}\left(t^{\prime}\right)\right\|<\varepsilon$ whenever $\left(t, t^{\prime}\right) \in V$.

Given a net $\left\{v_{\alpha}\right\} \subset \mathscr{M}_{t}^{+}(T, Y)$ and a $\tau$-smooth measure $\nu \in \mathscr{M}_{t}^{+}(T, Y)$, if $\lim _{\alpha} \int_{T} g d \nu_{\alpha}=\int_{T} g d v$ for every $g \in U(T)$, then $\lim _{\alpha} \int_{T} \varphi_{\alpha} \widetilde{\otimes} d \nu_{\alpha}=\int_{T} \varphi \widetilde{\otimes} d \nu$.

To prove Theorem 4.1, we need several auxiliary results. In what follows, for any $V \in \mathscr{U}_{T}$ and $t \in T$, put $V(t)=\left\{t^{\prime} \in T:\left(t, t^{\prime}\right) \in V\right\}$.

LEMMA 4.2. Let $T$ be a uniform space with the uniformity $\mathscr{U}_{T}$. Let $Y$ be a Banach space. Assume that $\nu \in \mathscr{M}(T, Y)$ is $\tau$-smooth. Then, for each $\varepsilon>0$ and $V \in \mathscr{U}_{T}$, we can find a finite subset $\left\{t_{1}, t_{2}, \ldots, t_{n}\right\}$ of $T$ and $h \in U(T)$ with $0 \leq h \leq 1$ satisfying the following conditions:

(i) $h\left(t_{i}\right)=0$ for all $i=1,2, \ldots, n ; h(t)=1$ for all $t \notin \bigcup_{i=1}^{n} V\left(t_{i}\right)$, and

(ii) $\left\|\int_{T} h d \nu\right\|<\varepsilon$.

Proof. Let $V \in \mathscr{U}_{T}$ and fix $\varepsilon>0$. Then, for each $a \in T$, we can find $d_{a} \in U(T)$ satisfying $0 \leq d_{a} \leq 1, d_{a}(a)=0$, and $d_{a}(t)=1$ if $t \notin V(a)$ (the existence of such a function $d_{a}$ follows from a proof of Uniformizable Theorem [13, Proposition 11.5]).

Let $\alpha$ range over the finite subsets of $T$ and for $\alpha=\left\{t_{1}, t_{2}, \ldots, t_{n}\right\}$, put $h_{\alpha}(t)=$ $\min _{1 \leq i \leq n} d_{t_{i}}(t)$ for $t \in T$. Then, $\left\{h_{\alpha}\right\}$ is a decreasing net in $U(T)$ converging pointwise to 0 (the ordering for the $\alpha$ 's being the set-theoretical inclusion). Since $\nu$ is $\tau$-smooth, by Proposition 2.1, we have $\left\|\int_{T} h_{\alpha} d \nu\right\|<\varepsilon$ for some $\alpha=\left\{t_{1}, t_{2}, \ldots, t_{n}\right\}$, and this function $h_{\alpha}$ is a required one.

PROPOSITION 4.3. Let $T$ be a uniform space with the uniformity $\mathscr{U}_{T}$. Let $X$ be a $B a$ nach space and $Y$ a Banach iattice. Let $\left\{\nu_{\alpha}\right\}$ be a net in $\mathscr{M}^{+}(T, Y)$ and $\nu \in \mathscr{M}^{+}(T, Y)$. Assume that $v$ is tight. Then the following two conditions are equivalent: 
(i) For every $g \in U(T)$, we have

$$
\int_{T} g d \nu_{\alpha} \rightarrow \int_{T} g d \nu
$$

(ii) For every $\varphi \in U(T, X)$, we have

$$
\sup _{x^{*} \in B_{X^{*}}}\left\|\int_{T} x^{*} \varphi d \nu_{\alpha}-\int_{T} x^{*} \varphi d \nu\right\| \rightarrow 0 .
$$

ProOF. It is routine to prove that (ii) implies (i), and hence we prove (i) implies (ii).

Fix $\varphi \in U(T, X)$, and we may assume that $\|\varphi\|_{\infty} \leq 1$ without loss of generality, since we obtain the result for a general $\varphi$ by considering $\varphi /\|\varphi\|_{\infty}$ in place of $\varphi$.

Given $\varepsilon>0$, the tight measure $\nu$ satisfies $\|v\|(T-K)<\varepsilon$ for some compact subset $K$ of $T$. We first claim that there exists an open set $V \in \mathscr{U}_{T}$ such that the inequality

$$
\sup _{t \in K(V)}\left|u^{*} \varphi(t)-v^{*} \varphi(t)\right| \leq 3 \varepsilon
$$

holds for all $u^{*}, v^{*} \in \boldsymbol{B}_{X^{*}}$ satisfying

$$
\sup _{t \in K}\left|u^{*} \varphi(t)-v^{*} \varphi(t)\right|<\varepsilon,
$$

where $K(V)=\left\{t \in T:\left(t, t^{\prime}\right) \in V\right.$ for some $\left.t^{\prime} \in K\right\}$. In fact, there exists an open set $V \in \mathscr{U}_{T}$ such that $\sup _{x^{*} \in B_{X^{*}}}\left|x^{*} \varphi(t)-x^{*} \varphi\left(t^{\prime}\right)\right|=\left\|\varphi(t)-\varphi\left(t^{\prime}\right)\right\|<\varepsilon$ whenever $\left(t, t^{\prime}\right) \in V$. Take elements $u^{*}, v^{*} \in B_{X}$. such that (4.4) holds. Let $t \in K(V)$ and take $t^{\prime} \in K$ satisfying $\left(t, t^{\prime}\right) \in V$. Then, we have

$$
\begin{aligned}
\left|u^{*} \varphi(t)-v^{*} \varphi(t)\right| \leq & \left|u^{*} \varphi(t)-u^{*} \varphi\left(t^{\prime}\right)\right|+\left|u^{*} \varphi\left(t^{\prime}\right)-v^{*} \varphi\left(t^{\prime}\right)\right| \\
& +\left|v^{*} \varphi\left(t^{\prime}\right)-v^{*} \varphi(t)\right|<3 \varepsilon,
\end{aligned}
$$

which implies (4.3).

Next we claim that

$$
\limsup _{\alpha}\left\|v_{\alpha}\right\|(T-K(V))<\varepsilon
$$

Indeed, since $K(V)$ is an open subset containing the compact set $K$, there exists $g \in U(T)$ vanishing on $K$ such that $0 \leq g \leq 1$ and $g=1$ on $T-K(V)$ (adapt the proof of [13, Proposition 11.5]). The positive measures $\nu_{\alpha}$ and $\nu$ satisfy $\left\|\nu_{\alpha}\right\|(T-K(V)) \leq$ $\left\|\int_{T} g d \nu_{\alpha}\right\|$ and $\left\|\int_{T} g d v\right\| \leq\|\nu\|(T-K)$. It follows from assumption (i) applied 
to $g \in U(T)$ that $\left\|\int_{T} g d v_{\alpha}\right\| \rightarrow\left\|\int_{T} g d \nu\right\|$. Hence, $\lim \sup _{\alpha}\left\|\nu_{\alpha}\right\|(T-K(V)) \leq$ $\|v\|(T-K)<\varepsilon$, which establishes (4.5).

Now, the set of functions $\left\{x^{*} \varphi: x^{*} \in B_{X^{*}}\right\}$ restricted to $K$ is uniformly bounded and uniformly equicontinuous in $C(K)$, so that it is a relatively compact subset of $C(K)$ by the Arzelà-Ascoli theorem. In other words, there exists a finite subset $B_{0}$ of $B_{X}$. such that for any $x^{*} \in B_{X^{*}}$ there is $x_{0}^{*} \in B_{0}$ with $\sup _{t \in K}\left|x^{*} \varphi(t)-x_{0}^{*} \varphi(t)\right|<\varepsilon$, and hence we have $\sup _{t \in K(V)}\left|x^{*} \varphi(t)-x_{0}^{*} \varphi(t)\right| \leq 3 \varepsilon$ by (4.3). Let $x^{*} \in B_{X^{*}}$ and take $x_{0}^{*} \in B_{0}$ as above. Then

$$
\begin{aligned}
& \left\|\int_{T} x^{*} \varphi d v_{\alpha}-\int_{T} x^{*} \varphi d \nu\right\| \\
& \leq\left\|\int_{T}\left|x^{*} \varphi-x_{0}^{*} \varphi\right| d \nu_{\alpha}\right\|+\max _{u^{*} \in B_{0}}\left\|\int_{T} u^{*} \varphi d \nu_{\alpha}-\int_{T} u^{*} \varphi d \nu\right\| \\
& \quad+\left\|\int_{T}\left|x_{0}^{*} \varphi-x^{*} \varphi\right| d \nu\right\| .
\end{aligned}
$$

Further, we have

$$
\begin{aligned}
\left\|\int_{T}\left|x^{*} \varphi-x_{0}^{*} \varphi\right| d \nu_{\alpha}\right\| \leq & \left\|\int_{T-K(V)}\left|x^{*} \varphi-x_{0}^{*} \varphi\right| d \nu_{\alpha}\right\| \\
& +\left\|\int_{K(V)}\left|x^{*} \varphi-x_{0}^{*} \varphi\right| d \nu_{\alpha}\right\| \\
& \leq 2\left\|v_{\alpha}\right\|(T-K(V))+3 \varepsilon\left\|v_{\alpha}(T)\right\|
\end{aligned}
$$

and

$$
\left\|\int_{T}\left|x_{0}^{*} \varphi-x^{*} \varphi\right| d \nu\right\| \leq 2\|\nu\|(T-K)+\varepsilon\|\nu(T)\|<2 \varepsilon+\varepsilon\|\nu(T)\| .
$$

It follows from (4.6)-(4.8) that

$$
\begin{aligned}
\sup _{x^{*} \in B_{X^{*}}} & \left\|\int_{T} x^{*} \varphi d v_{\alpha}-\int_{T} x^{*} \varphi d \nu\right\| \\
\leq & 2\left\|\nu_{\alpha}\right\|(T-K(V))+3 \varepsilon\left\|v_{\alpha}(T)\right\|+2 \varepsilon+\varepsilon\|\nu(T)\| \\
& \quad+\max _{u^{*} \in B_{0}}\left\|\int_{T} u^{*} \varphi d v_{\alpha}-\int_{T} u^{*} \varphi d \nu\right\| .
\end{aligned}
$$

Now an appeal to (4.1) gives $\left\|\nu_{\alpha}(T)\right\|=\left\|\int_{T} 1 d \nu_{\alpha}\right\| \rightarrow\left\|\int_{T} 1 d \nu\right\|=\|\nu(T)\|$ and

$$
\max _{u^{*} \in B_{0}}\left\|\int_{T} u^{*} \varphi d v_{\alpha}-\int_{T} u^{*} \varphi d \nu\right\| \rightarrow 0
$$


and hence by (4.5) and (4.9) we have

$$
\begin{aligned}
\underset{\alpha}{\limsup } & \left\{\sup _{x^{*} \in B_{X^{*}}}\left\|\int_{T} x^{*} \varphi d v_{\alpha}-\int_{T} x^{*} \varphi d \nu\right\|\right\} \\
& \leq \underset{\alpha}{2 \limsup }\left\|\nu_{\alpha}\right\|(T-K(V))+3 \varepsilon\|\nu(T)\|+2 \varepsilon+\varepsilon\|\nu(T)\|^{\circ} \\
& <4 \varepsilon+4 \varepsilon\|\nu(T)\|=4 \varepsilon(1+\|\nu(T)\|),
\end{aligned}
$$

which establishes (4.2).

PROPOSITION 4.4. Let $T$ be a uniform space. Let $X$ be a Banach space and $Y$ a Banach lattice. Let $\left\{\nu_{\alpha}\right\}$ be a net in $\mathscr{M}_{t}^{+}(T, Y)$ and $\nu \in \mathscr{M}_{t}^{+}(T, Y)$. Then the following two conditions are equivalent:

(i) For every $g \in U(T)$, we have $\int_{T} g d v_{\alpha} \rightarrow \int_{T} g d \nu$.

(ii) For every $\varphi \in U(T, X)$, we have $\int_{T} \varphi \widetilde{\otimes} d \nu_{\alpha} \rightarrow \int_{T} \varphi \widetilde{\otimes} d \nu$.

Proof. It is obvious that (ii) implies (i), and hence we prove (i) implies (ii).

Fix $\varphi \in U(T, X)$. Then, $\sup _{x^{*} \in B_{X^{*}}}\left\|\int_{T} x^{*} \varphi d \nu_{\alpha}-\int_{T} x^{*} \varphi d v\right\| \rightarrow 0$ by Proposition 4.3. It follows from Proposition 3.3 that $\varphi$ is integrable with respect to both $\nu_{\alpha}$ and $v$. Hence, by $[11$, page 327$]$ we have

$$
\begin{aligned}
\left\|\int_{T} \varphi \tilde{\otimes} d \nu_{\alpha}-\int_{T} \varphi \widetilde{\otimes} d \nu\right\| & =\sup _{\substack{x^{*} B_{X^{*}} \\
y^{*} \in B_{Y^{*}}}}\left|\int_{T} x^{*} \varphi d\left(y^{*} v_{\alpha}\right)-\int_{T} x^{*} \varphi d\left(y^{*} \nu\right)\right| \\
& =\sup _{x^{*} \in B_{X^{*}}}\left\|\int_{T} x^{*} \varphi d \nu_{\alpha}-\int_{T} x^{*} \varphi d \nu\right\| \rightarrow 0,
\end{aligned}
$$

which establishes (ii).

Proof of ThEOREM 4.1. Fix $\varepsilon>0$. By assumption (iii) we can find $V \in \mathscr{U}_{T}$ such that

$$
\sup _{\alpha}\left\|\varphi_{\alpha}(t)-\varphi_{\alpha}\left(t^{\prime}\right)\right\|<\varepsilon \quad \text { and } \quad\left\|\varphi(t)-\varphi\left(t^{\prime}\right)\right\|<\varepsilon
$$

whenever $\left(t, t^{\prime}\right) \in V$. Then, Proposition 3.3 allows us to define continuous linear operators $L_{\alpha}, L: U(T, X) \rightarrow X \widetilde{\otimes} Y$ by $L_{\alpha} \psi=\int_{T} \psi \widetilde{\otimes} d v_{\alpha}$ and $L \psi=\int_{T} \psi \widetilde{\otimes} d v$, $\psi \in U(T, X)$. Then the equality $L_{\alpha} \varphi_{\alpha}=L_{\alpha} \varphi+L_{\alpha}\left(\varphi_{\alpha}-\varphi\right)$ holds.

We first prove that

$$
\left\|L_{\alpha}\left(\varphi_{\alpha}-\varphi\right)\right\| \rightarrow 0
$$


To this end, take a finite subset $\left\{t_{1}, t_{2}, \ldots, t_{n}\right\}$ of $T$ and $h \in U(T)$ with $0 \leq h \leq 1$ satisfying (i) and (ii) of Lemma 4.2. Then, assumption (i) of Theorem 4.1 ensures that

$$
\lim _{\alpha} \max _{1 \leq i \leq n}\left\|\varphi_{\alpha}\left(t_{i}\right)-\varphi\left(t_{i}\right)\right\|=0
$$

We claim that

$$
\limsup _{\alpha}\left\|(1-h)\left(\varphi_{\alpha}-\varphi\right)\right\|_{\infty} \leq 2 \varepsilon
$$

In fact, let $t \in \bigcup_{i=1}^{n} V\left(t_{i}\right)$. Then, there exists $i_{0}$ with $1 \leq i_{0} \leq n$ such that $\left(t_{i_{0}}, t\right) \in V$. Hence, by (4.10) we have $\sup _{t \in \bigcup_{i=1}^{n} v\left(t_{i}\right)}\left\|\varphi_{\alpha}(t)-\varphi(t)\right\| \leq 2 \varepsilon+\max _{1 \leq i \leq n}\left\|\varphi_{\alpha}\left(t_{i}\right)-\varphi\left(t_{i}\right)\right\|$ for each $\alpha$. Thus, by (4.12) we have $\lim \sup _{\alpha} \sup _{t \in \bigcup_{i=1}^{n} v\left(t_{i}\right)}\left\|\varphi_{\alpha}(t)-\varphi(t)\right\| \leq 2 \varepsilon$. On the other hand, since the function $h$ satisfies (i) of Lemma 4.2, we have

$$
\left\|(1-h)\left(\varphi_{\alpha}-\varphi\right)\right\|_{\infty} \leq \sup _{t \in \bigcup_{i=1}^{n} v\left(t_{i}\right)}\left\|\varphi_{\alpha}(t)-\varphi(t)\right\|
$$

for all $\alpha$, which implies (4.13).

Now, observe that $\left\|L_{\alpha}\left(\varphi_{\alpha}-\varphi\right)\right\| \leq\left\|L_{\alpha}\left(h\left(\varphi_{\alpha}-\varphi\right)\right)\right\|+\left\|L_{\alpha}\left((1-h)\left(\varphi_{\alpha}-\varphi\right)\right)\right\|$ for each $\alpha$. Recalling assumption (ii), we put $M=\sup _{\alpha}\left\|\varphi_{\alpha}\right\|_{\infty}<\infty$. It follows from Proposition 3.2 that

$$
\left\|L_{\alpha}\left(h\left(\varphi_{\alpha}-\varphi\right)\right)\right\| \leq\left\|\varphi_{\alpha}-\varphi\right\|_{\infty} \cdot\left\|\int_{T} h d v_{\alpha}\right\| \leq\left(M+\|\varphi\|_{\infty}\right)\left\|\int_{T} h d v_{\alpha}\right\| .
$$

On the other hand, it follows from [1, Theorem 4] that

$$
\left\|L_{\alpha}\left((1-h)\left(\varphi_{\alpha}-\varphi\right)\right)\right\| \leq\left\|(1-h)\left(\varphi_{\alpha}-\varphi\right)\right\|_{\infty} \cdot\left\|\nu_{\alpha}\right\|(T) .
$$

Consequently, for each $\alpha$ we have

$$
\left\|L_{\alpha}\left(\varphi_{\alpha}-\varphi\right)\right\| \leq\left(M+\|\varphi\|_{\infty}\right)\left\|\int_{T} h d v_{\alpha}\right\|+\left\|(1-h)\left(\varphi_{\alpha}-\varphi\right)\right\|_{\infty}\left\|\nu_{\alpha}\right\|(T) .
$$

By the assumption of Theorem 4.1, we have $\lim _{\alpha} \int_{T} h d \nu_{\alpha}=\int_{T} h d \nu$ and

$$
\lim _{\alpha}\left\|\nu_{\alpha}\right\|(T)=\lim _{\alpha}\left\|v_{\alpha}(T)\right\|=\|v(T)\|=\|v\|(T) .
$$

Thus, it follows from (4.13) and (4.14) that

$$
\begin{aligned}
& \underset{\alpha}{\limsup }\left\|L_{\alpha}\left(\varphi_{\alpha}-\varphi\right)\right\| \leq\left(M+\|\varphi\|_{\infty}\right)\left\|\int_{T} h d \nu\right\|+2 \varepsilon\|v\|(T) \\
& <\left(M+\|\varphi\|_{\infty}\right) \varepsilon+2 \varepsilon\|\nu\|(T)
\end{aligned}
$$

which establishes (4.11).

By Proposition 4.4 and assumptions of Theorem 4.1 we have $\left\|L_{\alpha} \varphi-L \varphi\right\| \rightarrow 0$. This together with (4.11) implies $\left\|L_{\alpha} \varphi_{\alpha}-L \varphi\right\| \rightarrow 0$ and the proof is complete. 


\section{Weak convergence of injective tensor product measures}

We first recall the definition of weak convergence of vector measures. Let $S$ be a topological space and $X$ a Banach space. Let $\left\{\mu_{\alpha}\right\}$ be a net in $\mathscr{M}(S, X)$ and $\mu \in \mathscr{M}(S, X)$. We say that $\left\{\mu_{\alpha}\right\}$ converges weakly to $\mu$, and write $\mu_{\alpha} \stackrel{\cdot w}{\longrightarrow} \mu$, if for each $f \in C(S)$ we have $\lim _{\alpha} \int_{S} f d \mu_{\alpha}=\int_{S} f d \mu$ in the norm of $X$; see [6, 22].

The following proposition asserts that in the case of positive vector measures, weak convergence follows from the validity of the above convergence for only bounded uniformly continuous functions $f$ on $S$; see for example [23, Theorem 8.1 (the Portmanteau Theorem)].

PROPOSITION 5.1. Let $S$ be a uniform space with the uniformity $\mathscr{U}_{S}$. Let $X$ be a Banach lattice. Let $\left\{\mu_{\alpha}\right\}$ be a net in $\mathscr{M}^{+}(S, X)$ and $\mu \in \mathscr{M}^{+}(S, X)$. Assume that $\mu$ is tight. Then the following two conditions are equivalent:

(i) For every $f \in U(S)$, we have $\int_{S} f d \mu_{\alpha} \rightarrow \int_{S} f d \mu$.

(ii) For every $f \in C(S)$, we have $\int_{S} f d \mu_{\alpha} \rightarrow \int_{S} f d \mu$.

ProOF. It is obvious that (ii) implies (i), and hence we prove (i) implies (ii).

Fix $\varepsilon>0$ and $f \in C(S)$. Then, the tight measure $\mu$ satisfies $\|\mu\|(S-K)<\varepsilon$ for some compact subset $K$ of $S$, and it follows from [21, Lemma V.1, page 250] that there exists an open, symmetric set $V \in \mathscr{U}_{S}$ satisfying

$$
\left|f(s)-f\left(s^{\prime}\right)\right|<\varepsilon \quad \text { whenever }\left(s, s^{\prime}\right) \in V \text { and } s^{\prime} \in K .
$$

Further, there is a function $g \in U(S)$ such that $g(s)=f(s)$ for all $s \in K$ and $\|g\|_{\infty}=\sup _{s \in K}|f(s)| ;$ see $[4$, Chapter IX, Exercises, Section 1, no. 22]. Then, there exists an open, symmetric set $V^{\prime} \in \mathscr{U}_{S}$ such that

$$
\left|g(s)-g\left(s^{\prime}\right)\right|<\varepsilon \quad \text { whenever }\left(s, s^{\prime}\right) \in V^{\prime} .
$$

Put $W=V \cap V^{\prime}$. Then, $W$ is also an open, symmetric subset of $\mathscr{U}_{S}$, and (5.1) and (5.2) remain true for the set $W$ instead of $V$ and $V^{\prime}$, respectively.

Put $K(W)=\left\{s \in S:\left(s, s^{\prime}\right) \in W\right.$ for some $\left.s^{\prime} \in K\right\}$. Then, by similar arguments as in the proof of (4.3) and (4.5) of Proposition 4.3, it follows from (5.1), (5.2), the positivity of $\mu$ and assumption (i) that

$$
\sup _{s \in K(W)}|f(s)-g(s)| \leq 2 \varepsilon \quad \text { and } \quad \limsup _{\alpha}\left\|\mu_{\alpha}\right\|(S-K(W)) \leq \varepsilon .
$$

Now, for each $\alpha$ we have

$$
\begin{aligned}
\left\|\int_{S} f d \mu_{\alpha}-\int_{S} f d \mu\right\| \leq & \left\|\int_{S} f d \mu_{\alpha}-\int_{S} g d \mu_{\alpha}\right\|+\left\|\int_{S} g d \mu_{\alpha}-\int_{S} g d \mu\right\| \\
& +\left\|\int_{S} g d \mu-\int_{S} f d \mu\right\|
\end{aligned}
$$


By the first inequality of (5.3), for each $\alpha$ we have

$$
\begin{aligned}
& \left\|\int_{S} f d \mu_{\alpha}-\int_{S} g d \mu_{\alpha}\right\| \\
& \quad \leq\left\|\int_{S-K(W)} f d \mu_{\alpha}\right\|+\left\|\int_{S-K(W)} g d \mu_{\alpha}\right\|+\left\|\int_{K(W)}(f-g) d \mu_{\alpha}\right\| \\
& \quad \leq\|f\|_{\infty}\left\|\mu_{\alpha}\right\|(S-K(W))+\|g\|_{\infty}\left\|\mu_{\alpha}\right\|(S-K(W))+2 \varepsilon\left\|\mu_{\alpha}\right\|(S) .
\end{aligned}
$$

Similarly, noting that $\|\mu\|(S-K)<\varepsilon$ and $g=f$ on $K$, for each $\alpha$ we have

$$
\left\|\int_{S} g d \mu-\int_{S} f d \mu\right\| \leq \varepsilon\left(\|f\|_{\infty}+\|g\|_{\infty}\right) .
$$

On the other hand, it follows from assumption (i) that $\lim _{\alpha}\left\|\mu_{\alpha}\right\|(S)=\lim _{\alpha}\left\|\mu_{\alpha}(S)\right\|=$ $\|\mu(S)\|=\|\mu\|(S)$ and $\lim _{\alpha} \int_{S} g d \mu_{\alpha}=\int_{S} g d \mu$. This together with (5.3)-(5.6) implies $\lim \sup _{\alpha}\left\|\int_{S} f d \mu_{\alpha}-\int_{S} f d \mu\right\| \leq 2 \varepsilon\left(\|f\|_{\infty}+\|g\|_{\infty}+\|\mu\|(S)\right)$, which establishes (ii).

LEMMA 5.2. Let $S$ and $T$ be uniform spaces with the uniformities $\mathscr{U}_{S}$ and $\mathscr{U}_{T}$, respectively. Let $X$ be a Banach space. Assume that a net $\left\{\mu_{\alpha}\right\}$ in $\mathscr{M}(S, X)$ is uniformly bounded, that is, $\sup _{\alpha}\left\|\mu_{\alpha}\right\|(S)<\infty$. Let $h \in U(S \times T)$ and put $\varphi_{\alpha}(t)=$ $\int_{S} h(s, t) \mu_{\alpha}(d s)$ for all $\alpha$ and $t \in T$. Then, $\left\{\varphi_{\alpha}\right\}$ satisfies assumptions (ii) and (iii) of Theorem 4.1.

Proof. The uniform boundedness of $\left\{\varphi_{\alpha}\right\}$ follows from [2, Theorem 2.6].

Put $M=\sup _{\alpha}\left\|\mu_{\alpha}\right\|(S)<\infty$. Given $\varepsilon>0$, chose $V \in \mathscr{U}_{S}$ and $W \in \mathscr{U}_{T}$ such that $\left|h(s, t)-h\left(s^{\prime}, t^{\prime}\right)\right|<\varepsilon /(M+1)$ whenever $\left(s, s^{\prime}\right) \in V$ and $\left(t, t^{\prime}\right) \in W$. Thus, if $\left(t, t^{\prime}\right) \in W$, then for all $\alpha$ we have

$$
\begin{aligned}
\left\|\varphi_{\alpha}(t)-\varphi_{\alpha}\left(t^{\prime}\right)\right\| & =\left\|\int_{S}\left(h(s, t)-h\left(s, t^{\prime}\right)\right) \mu_{\alpha}(d s)\right\| \\
& \leq \frac{\varepsilon}{M+1} \cdot\left\|\mu_{\alpha}\right\|(S) \leq \frac{\varepsilon}{M+1} \cdot M<\varepsilon,
\end{aligned}
$$

which implies the uniform equicontinuity of $\left\{\varphi_{\alpha}\right\}$.

Let $S$ and $T$ be uniform spaces. Let $X$ and $Y$ be Banach lattices. Then, by [8, Theorem], given vector measures $\mu \in \mathscr{M}(S, X)$ and $\nu \in \mathscr{M}(T, Y)$, there exists a unique vector measure $\mu \widetilde{\otimes} \nu: \mathscr{B}(S) \times \mathscr{B}(T) \rightarrow X \widetilde{\otimes} Y$, which is called the injective tensor product of $\mu$ and $\nu$, such that $(\mu \widetilde{\otimes} \nu)(A \times B)=\mu(A) \otimes \nu(B)$ for all $A \in \mathscr{B}(S)$ and $B \in \mathscr{B}(T)$; see also [16, Theorem]. In this section, as an application of Theorem 4.1, we consider a problem of joint continuity of the injective tensor product of positive vector measures in certain Banach lattices. 
In the rest of this paper, we assume that $S$ and $T$ satisfy $\mathscr{B}(S \times T)=\mathscr{B}(S) \times \mathscr{B}(T)$ (it is routine to check that this condition is satisfied, for instance, either $S$ or $T$ has a countable base of open sets). In this case, we can view the injective tensor product $\mu \widetilde{\otimes} \nu$ as a vector measure defined on $\mathscr{B}(S \times T)$, and integrate any (uniformly) continuous real function with respect to $\mu \widetilde{\otimes} \nu$. As an application of Theorem 4.1 we have the following result.

THEOREM 5.3. Let $S$ and $T$ be uniform spaces. Let $X$ and $Y$ be Banach lattices. Let $\left\{\mu_{\alpha}\right\}$ be a net in $\mathscr{M}^{+}(S, X)$ and $\mu \in \mathscr{M}^{+}(S, X)$. Let $\left\{\nu_{\alpha}\right\}$ be a net in $\mathscr{M}_{t}^{+}(T, Y)$ and $\nu \in \mathscr{M}_{t}^{+}(T, Y)$. Assume that $\nu$ is $\tau$-smooth. If $\int_{S} f d \mu_{\alpha} \rightarrow \int_{S} f d \mu$ and $\int_{T} g d \nu_{\alpha} \rightarrow \int_{T} g d v$ for every $f \in U(S)$ and $g \in U(T)$, then $\int_{S \times T} h d\left(\mu_{\alpha} \widetilde{\otimes} v_{\alpha}\right) \rightarrow$ $\int_{S \times T} h d(\mu \widetilde{\otimes} \nu)$ for every $h \in U(S \times T)$.

PROOF. We may assume without loss of generality that $\left\{\mu_{\alpha}\right\}$ and $\left\{v_{\alpha}\right\}$ are uniformly bounded, that is, $\sup _{\alpha}\left\|\mu_{\alpha}\right\|(S)<\infty$ and $\sup _{\alpha}\left\|v_{\alpha}\right\|(T)<\infty$.

Fix $h \in U(S \times T)$, and put $\varphi_{\alpha}(t)=\int_{S} h(s, t) \mu_{\alpha}(d s)$ and $\varphi(t)=\int_{S} h(s, t) \mu(d s)$ for each $\alpha$ and $t \in T$. Then, by Lemma 5.2 and the assumptions of this theorem, $\left\{\varphi_{\alpha}\right\}$ and $\varphi$ satisfy the conditions of Theorem 4.1, and hence we have $\int_{T} \varphi_{\alpha} \widetilde{\otimes} d \nu_{\alpha} \rightarrow$ $\int_{T} \varphi \widetilde{\otimes} d \nu$. By the Fubini theorem for injective tensor product measures (see, for instance, [11, Theorem 13]), we have $\int_{T} \varphi_{\alpha} \widetilde{\otimes} d v_{\alpha}=\int_{S \times T} h d\left(\mu_{\alpha} \widetilde{\otimes} v_{\alpha}\right)$ and $\int_{T} \varphi \widetilde{\otimes} d \nu=\int_{S \times T} h d(\mu \widetilde{\otimes} \nu)$, which proves the theorem.

Let $X$ and $Y$ be Banach lattices. Then, in general, the injective tensor product $X \widetilde{\otimes} Y$ or the projective tensor product may not be a vector lattice for the natural ordering. However, the injective tensor products of some important examples of Banach lattices are also Banach lattices; see the examples after the proof of the following theorem.

Let $X$ and $Y$ be Banach lattices such that the injective tensor product $X \widetilde{\otimes} Y$ is also a Banach lattice satisfying the condition $x \otimes y \geq 0$ for every $x \geq 0$ and $y \geq 0$. Let $(\Omega, \mathscr{A})$ and $(\Gamma, \mathscr{B})$ be measurable spaces. Let $\mu: \mathscr{A} \rightarrow X$ and $\nu: \mathscr{B} \rightarrow Y$ be vector measures. Then it is easy to verify that if $\mu$ and $\nu$ are positive, so is the injective tensor product $\mu \widetilde{\otimes} \nu$. In this case, we have an affirmative answer for a problem of joint continuity of the injective tensor products with respect to the weak convergence of vector measures.

THEOREM 5.4. Let $S$ and $T$ be uniform spaces. Let $X$ and $Y$ be Banach lattices such that the injective tensor product $X \widetilde{\otimes} Y$ is also a Banach lattice satisfying the condition $x \otimes y \geq 0$ for every $x \geq 0$ and $y \geq 0$. Let $\left\{\mu_{\alpha}\right\}$ be a net in $\mathscr{M}^{+}(S, X)$ and $\mu \in \mathscr{M}_{t}^{+}(S, X)$. Let $\left\{\nu_{\alpha}\right\}$ be a net in $\mathscr{M}_{t}^{+}(T, Y)$ and $\nu \in \mathscr{M}_{t}^{+}(T, Y)$. Assume that $\nu$ is $\tau$-smooth. If $\mu_{\alpha} \stackrel{w}{\longrightarrow} \mu$ and $v_{\alpha} \stackrel{w}{\longrightarrow} v$, then $\mu_{\alpha} \widetilde{\otimes} v_{\alpha} \stackrel{w}{\longrightarrow} \mu \widetilde{\otimes} v$.

PRoof. By Theorem 5.3, for every $h \in U(S \times T)$ we have $\int_{S \times T} h d\left(\mu_{\alpha} \widetilde{\otimes} v_{\alpha}\right) \rightarrow$ $\int_{S \times T} d(\mu \widetilde{\otimes} \nu)$. By assumption, $\mu_{\alpha} \widetilde{\otimes} \nu_{\alpha}$ 's and $\mu \widetilde{\otimes} \nu$ are positive, and it is easy 
to prove that $\mu \widetilde{\otimes} \nu$ is tight. Consequently, it follows from Proposition 5.1 that $\mu_{\alpha} \widetilde{\otimes} \nu_{\alpha} \stackrel{w}{\longrightarrow} \mu \widetilde{\otimes} \nu$.

REMARK. In the special case that $X=Y=\mathbb{R}$, an alternative proof of Theorem 5.4 is executed by a well-known criterion that one can prove the weak convergence of $\mu_{\alpha}$ to $\mu$ by showing that $\mu_{\alpha}(A) \rightarrow \mu(A)$ for some special class of sets $A$ (see, for instance, [24, Corollary 1 to Theorem I.3.5 and Proposition I.4.1]). However, it seems that the usual proof of the above criterion does not work well for positive vector measures, since the notions of limit infimum and limit supremum cannot be extended to general Banach lattices.

We finish this paper with examples of Banach lattices $X$ and $Y$ such that the injective tensor product $X \widetilde{\otimes} Y$ is also a Banach lattice satisfying the condition $x \otimes y \geq 0$ for every $x \geq 0$ and $y \geq 0$; see examples in [20, pages 274-276] and [12, page 90].

EXAMPLES. (1) If $K$ is a compact space and $Y$ be any Banach lattice, then $C(K) \widetilde{\otimes} Y$ is isometrically lattice isomorphic to the Banach lattice $C(K, Y)$. Especially, when $Y=C(L)$ for some compact space $L, C(K) \widetilde{\otimes} C(L)$ is isometrically lattice isomorphic to $C(K \times L)$.

(2) Let $P$ be a locally compact space and $Y$ be any Banach lattice. Denote by $C_{0}(P, Y)$ the Banach lattice with its canonical ordering of all continuous functions $\varphi: P \rightarrow Y$ such that for every $\varepsilon>0$, the set $\{s \in P:\|\varphi(s)\| \geq \varepsilon\}$ is compact. We write $C_{0}(P)=C_{0}(P, \mathbb{R})$. Then $C_{0}(P) \widetilde{\otimes} Y$ is isometrically lattice isomorphic to $C_{0}(P, Y)$. Especially, when $Y=C_{0}(Q)$ for some locally compact space $Q$, $C_{0}(P) \widetilde{\otimes} C_{0}(Q)$ is isometrically lattice isomorphic to $C_{0}(P \times Q)$.

(3) Let $(\Omega, \mathscr{A}, \sigma)$ be a measure space and $Y$ be any Banach lattice. Denote by $L^{\infty}(\Omega, Y)$ the Banach lattice of all (equivalence classes of) $\sigma$-essentially bounded measurable functions $\varphi: \Omega \rightarrow Y$ with its canonical ordering. We write $L^{\infty}(\Omega)=$ $L^{\infty}(\Omega, \mathbb{R})$. Then, $L^{\infty}(\Omega) \widetilde{\otimes} Y$ is a Banach lattice. However, in general, $L^{\infty}(\Omega) \widetilde{\otimes} Y$ is a proper closed subset of $L^{\infty}(\Omega, Y)$.

\section{Acknowledgement}

The author is very grateful to the referee for detailed comments and suggestions which have significantly improved this paper.

\section{References}

[1] R. G. Bartle, 'A general bilinear vector integral', Studia Math. 15 (1956), 337-352.

[2] R. G. Bartle, N. Dunford and J. Schwartz, 'Weak compactness and vector measures', Canad. J. Math. 7 (1955), 289-305. 
[3] P. Billingsley, Convergence of probability measures (Wiley, New York, 1968).

[4] N. Bourbaki, Topologie générale, 2nd edition (Hermann, Paris, 1958).

[5] I. Csiszár, 'On the weak* continuity of convolution in a convolution algebra over an arbitrary topological group', Studia Sci. Math. Hungar. 6 (1971), 27-40.

[6] M. Dekiert, Kompaktheit, Fortsetzbarkeit und Konvergenz von Vectormaßen (Dissertation, University of Essen, 1991).

[7] J. Diestel and J. J. Uhl, Jr., Vector measures, Amer. Math. Soc. Surveys 15 (Amer. Math. Soc., Providence, 1977).

[8] M. Duchoň and I. Kluvánek, 'Inductive tensor product of vector-valued measures', Mat. Časopis Sloven. Akad. Vied. 17 (1967), 108-112.

[9] R. M. Dudley, Real analysis and probability (Wadsworth \& Brooks/Cole, California, 1989).

[10] N. Dunford and J. T. Schwartz, Linear operators. Part 1: General theory (Wiley, New York, 1958).

[11] F. J. Freniche and J. C. García-Vázquez, 'The Bartle bilinear integration and Carleman operators', J. Math. Anal. Appl. 240 (1999), 324-339.

[12] A. Grothendieck, Produits tensoriels topologiques et espaces nucléaires, Mem. Amer. Math. Soc. 16 (Amer. Math. Soc., Providence, 1955).

[13] I. M. James, Topological and uniform spaces (Springer, New York, 1987).

[14] J. Kawabe, 'Weak convergence of tensor products of vector measures with values in nuclear spaces', Bull. Austral. Math. Soc. 59 (1999), 449-458.

[15] _ 'A type of Strassen's theorem for positive vector measures with values in dual spaces', Proc. Amer. Math. Soc. 128 (2000), 3291-3300.

[16] I. Kluvánek, 'On the product of vector measures', J. Austral. Math. Soc. 15 (1973), 22-26.

[17] I. Kluvánek and G. Knowles, Vector measures and control systems (North Holland, Amsterdam, 1975).

[18] D. R. Lewis, 'Integration with respect to vector measures', Pacific J. Math. 33 (1970), 157-165.

[19] M. März and R. M. Shortt, 'Weak convergence of vector measures', Publ. Math. Debrecen 45 (1994), 71-92.

[20] H. H. Schaefer, Banach lattices and positive operators (Springer, Berlin, 1974).

[21] L. Schwartz, Radon measures on arbitrary topological spaces and cylindrical measures (Tata Institute of Fundamental Research, Oxford University Press, 1973).

[22] R. M. Shortt, 'Strassen's theorem for vector measures', Proc. Amer. Math. Soc. 122 (1994), 811820 .

[23] F. Topsøe, Topology and measure, Lecture Notes in Math. 133 (Springer, New York, 1970).

[24] N. N. Vakhania, V. I. Tarieladze and S. A. Chobanyan, Probability distributions on Banach spaces (D. Reidel Publishing Company, 1987).

[25] M. Väth, Volterra and integral equations of vector functions (Marcel Dekker, New York, 2000).

[26] A. J. White, 'Convolution of vector measures', Proc. Roy. Soc. Edinburgh Sect. A 73 (1974/75), 117-135.

Department of Mathematics

Faculty of Engineering

Shinshu University

1-17-1 Wakasato

Nagano 380-8553

lapan

:-mail: jkawabe@gipwc.shinshu-u.ac.jp 
